\title{
Haptic Device that Presents Sensation Corresponding to Palm on Back of Hand for Teleoperation of Robot Hand Report2: Consideration on Decided Specification
}

\author{
Kyosuke Ushimaru, Noritaka Sato, Yoshifumi Morita \\ Department of Electrical and Mechanical Engineering, Graduate School of Engineering, \\ Nagoya Institute of Technology, Gokiso-cho, Showa-ku, Nagoya, Aichi 466-8555, Japan \\ E-mail: k.ushimaru.884@nitech.jp, sato.noritaka@nitech.ac.jp
}

\begin{abstract}
Recently, teleoperated rescue robots are required. However, it is known that the teleoperation of a robot hand mounted on a rescue robot is difficult. Therefore, we propose a new haptic device that presents haptic sensation for teleoperation of a robot hand. The device stimulates the back of the hand instead of the palm of the operator. Determination of required specifications by an experiment with subjects is written in this paper. To design the device, the interval of the stimulation points $(i)$, the diameter of the stimulation point $(d)$, and the force of the stimulation $(f)$ should be optimized. As a result of the experiment, we found that the accuracy rate is highest, when $(i, d, f)=(30 \mathrm{~mm}, 6 \mathrm{~mm}, 0.9 \mathrm{kgf})$. Moreover, we deeply considered on the decided specification with an additional experiment.
\end{abstract}

Keywords: Rescue robot, Haptic Device, Teleoperation, Robot Hand, Palm

\section{Introduction}

Since the Great Hanshin-Awaji Earthquake in 1995, research and development of disaster response robots have been conducted [1]. In recent years, there has been a demand for disaster response robots that can not only gather information but also perform tasks [2]. For this reason, disaster response robots equipped with robotic hands have begun to be developed so that they can work at disaster sites in the same manner as humans [3].

Since disaster response robots were operated in an unknown and extreme environment, they are often controlled by teleoperation [4]. In order to improve the efficiency of teleoperation, it is necessary to provide feedback to the operator on the state of contact between the fingers and palms of the robot hand. However, in the field of robot teleoperation, there are some systems that provide feedback on the contact state of the fingers [5][6], but we cannot find systems that provide feedback on the contact state of the palm.
In this study, we propose a method of substituting the contact state obtained from the palm of the robot hand to the back of the operator's hand. Okano et al. [9] have proposed a method of substituting the tactile presentation to other parts of the hand. However, Okano et al. substituted the sole of the foot for the tactile presentation of the whole hand. In contrast, this study substitutes the back of the hand for the tactile presentation of the hand. In the previous paper[11], we clarified the characteristic of the tactile sensation of the back of the hand in order to formulate the specifications required for the device that substitutes the back of the hand, as the first step of the research.

We prepared three candidates for each of the three types of stimulus points, i.e., the distance between the stimulus points, the diameter of the stimulus points, and the magnitude of the stimulus force. 


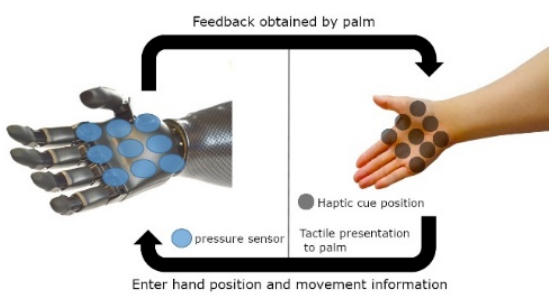

Fig. 1. Ideal method.

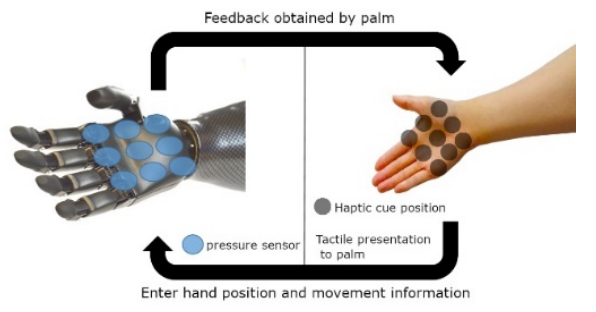

Fig. 2. Proposed method.

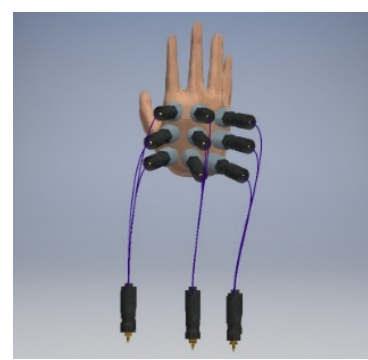

Fig. 3. Designed device with parameters which are obtained by experiment.

In this paper, we report the overview of the previous paper and the results of an additional experiment where the number of the stimulus points are one or two and the subjects wear gloves to verify whether the tactile sensation can be presented as a surface instead of a point, without changing the specifications.

\section{Proposed Device}

\subsection{Overview}

As shown in Fig. 1, it is ideal to present the tactile sensation on the palm of the hand in order to correctly convey the state of contact between the robot hand and the object to the operator. However, this method would interfere with the teleoperation of the robot hand, so we propose a method of presenting tactile sensations on the back of the hand as shown in Fig. 2. The following parameters are necessary to design the device:

- The interval between the stimulation points (interval: $i$ )

- $\quad$ The diameter of the stimulus point (diameter: $d$ )

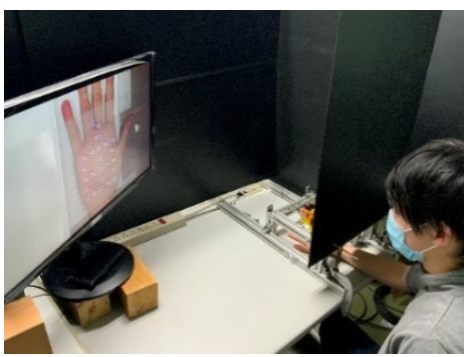

Fig. 4. Experimental scene.

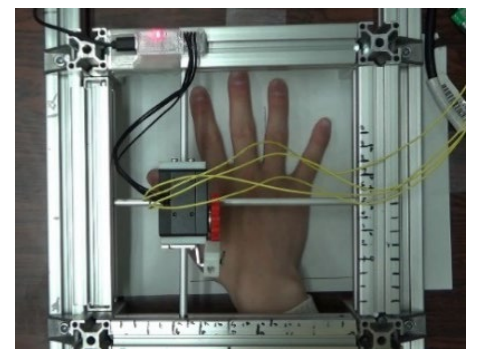

Fig. 5. Device for experiment.(Top view).

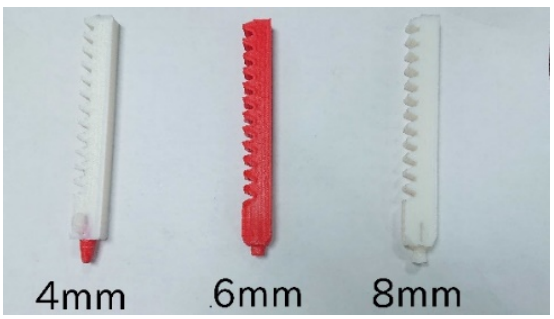

Fig. 6. Rod of experiment device

- The force of the stimulus (force: $f$ )

It is known that human skin sensation has a characteristic such as a two-point discrimination threshold.

Therefore, in the previous paper [11], we clarified the characteristics of the tactile sensation on the back of the human hand by subject experiments described in the next section and determined these specifications. Fig. 3 shows a conceptual view of the device designed by using the specifications determined by the experiments.

\subsection{Subject Experiment for Specification}

In this section, we report the optimal combination of the above parameters determined through a subject experiment. The procedure of the experiment is as follows: 10 subjects are prepared, and 10 points on the back of their hands are pressed one by one. The subjects were asked to answer where on the palm the pressed point corresponded to on the back of the hand. The interval of the stimulation points $(i)$, the diameter of the stimulation point $(d)$, and the force of the stimulation $(f)$ were the parameters of the combination. The combination with the highest ratio of correct answers is adopted as the required

(C) The 2021 International Conference on Artificial Life and Robotics (ICAROB2021), January 21 to 24, 2021 


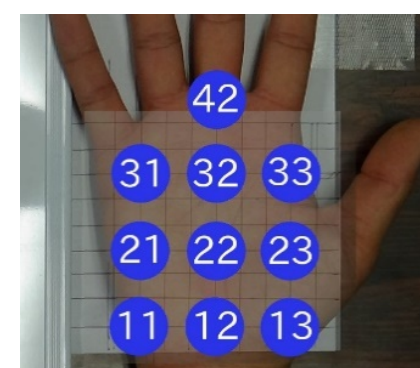

Fig. 7. Reference image for subject

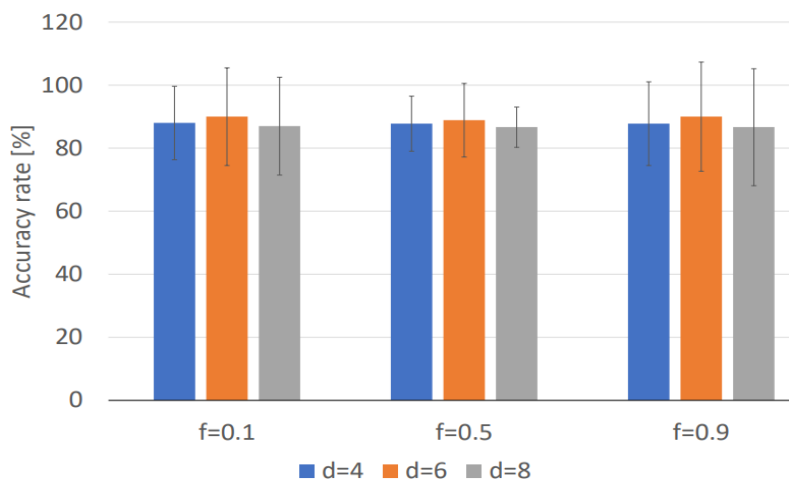

Fig. 8. Experimental result

specification for the device. The objective of the experiment is to find the combination with the highest correct ratio among 27 combinations, where the candidate values of $i$ are $10 \mathrm{~mm}, 20 \mathrm{~mm}$, and $30 \mathrm{~mm}, d$ is $4 \mathrm{~mm}, 6 \mathrm{~mm}$, and $8 \mathrm{~mm}$, and $f$ is $0.1 \mathrm{kgf}, 0.5 \mathrm{kgf}$, and 0.9 kgf. Fig. 4 shows the experimental scene. Figs. 5 and 6 show the devices for the experiment. Fig. 7 shows the image for the subject to answer the pressed place, which is displayed on the monitor.

\subsection{Experimental Results}

We collected a total of 2,700 data points from 10 subjects, each of whom pressed 10 points. A graph of the results for $i=30 \mathrm{~mm}$ is shown in Fig. 8. Note that other results are shown in our previous paper [11]. The vertical axis shows the average ratio of correct responses for each combination, and the error bars show the standard deviation of the ratio of correct responses. In the graph, three bars are grouped together when $d$ is the same. The blue, orange, and gray bars represent the cases where $f$ is $0.1 \mathrm{kgf}, 0.5 \mathrm{kgf}$, and $0.9 \mathrm{kgf}$, respectively.

The experimental results show that the correct response rate is highest, and the standard deviation is smallest when $(i, d, f)$ is $(30 \mathrm{~mm}, 6 \mathrm{~mm}, 0.9 \mathrm{kgf})$. In this case, the ratio of correct answers was $93 \%$ and the standard deviation was $7 \%$. Therefore, we decided to design the device using this combination since the mean value was highest and the variation was smallest.

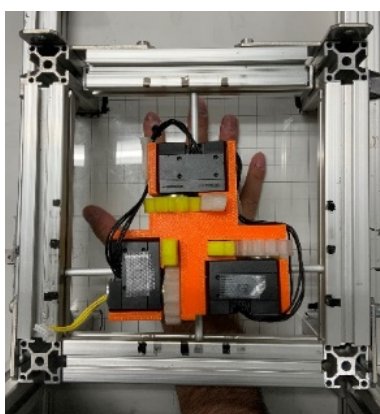

Fig. 9 Device for experiment.(Top view).

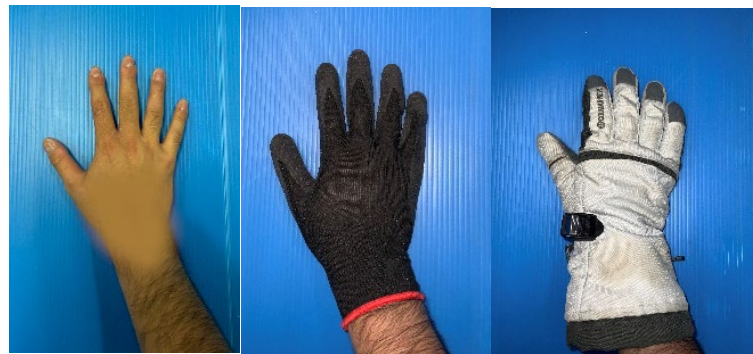

(1) Bare hand (2) Thin grove(3) Thick grove

Fig.10 Groves for experiment

\section{Experiment with Multiple Stimulation}

\subsection{Experimental Method}

In this section, we report a result of an experiment to check whether subjects misidentify the number of points by pressing the back of their hands simultaneously. In the experiment, we prepared three subjects, and asked them to press one or two points on the back of their hands and asked them to answer where the pressed points corresponded to in their palms. We use the combination $(i, d, f)=(30 \mathrm{~mm}, 6 \mathrm{~mm}, 0.9 \mathrm{~kg})$, which is the required specification for the device.

The subjects were three males (twenties), and in each condition, stimuli were applied to ten points on the back of the hand in a random order. The scene of the subject experiment is like the situation as shown in Fig. 4. We set up the experimental device which can press at two points simultaneously as shown in Fig. 9. The intervals of the points are fixed to $30 \mathrm{~mm}$. In this experiment, the subjects wear gloves to imagine the practical use as shown in Fig. 10. Fig. 11 shows the image for the subject to answer the pressed places.

\subsection{Results and Discussion}

We collected data for a total of 135 points, since 15 points were pressed by three subjects in three conditions. 


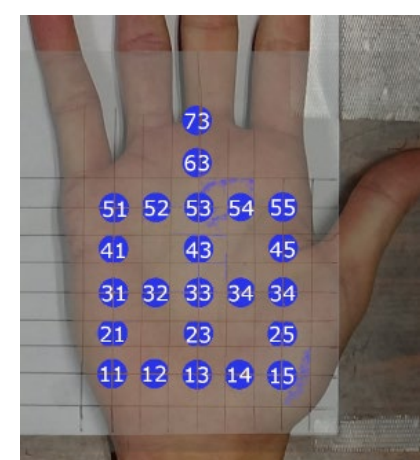

Fig.11 Reference image for subject

We calculated the average ratio of correct answers if the number and position of the points pressed corresponded to the points answered by the subjects. A graph of the results is shown in Fig. 12.

As a result of the experiment, subjects collectively recognized the positions of the pressed points similar to the previous experiment as shown in Fig. 8. Therefore, we think that the device which is developed with the specification obtained in the previous experiment may be effective in the case of multiple stimulations. On the other hand, we think that it is necessary to consider how to stimulate when one large object touches the robot hand. If we want the operator to recognize the tactile sensation as a surface, we may need to set the distance between the stimulus points to less than $30 \mathrm{~mm}$, which is the discrimination threshold of the back of the hand.

Moreover, the accuracy rate was decreased when the subjects wore thin gloves. Therefore, it is necessary to pay attention to the selection of the thickness and materials of the gloves. We think that it is interesting that the accuracy rates of some subjects are increased when the subjects wore thick gloves. We will verify this phenomenon with more subjects in the future.

\section{Conclusion}

In this study, we propose a method to present the contact state of the robot hand to the back of the operator's hand as a substitute for the palm in teleoperation of a disaster response robot. In this paper, we conducted an additional experiment with multiple stimulation.

In the future, we will conduct further verification of the experimental results. After that the device will be developed and the efficiency of teleoperation of the robot hand using the fabricated device will be verified.

\section{References}

1. S Tadokoro, et al.: "Rescue Robotics", Journal of the Robotics Society of Japan, Volume 19, Issue 6, Pages 685688, 2001. (In Japanese)

(C) The 2021 International Conference on Artificial Life and Robotics (ICAROB2021), January 21 to 24, 2021

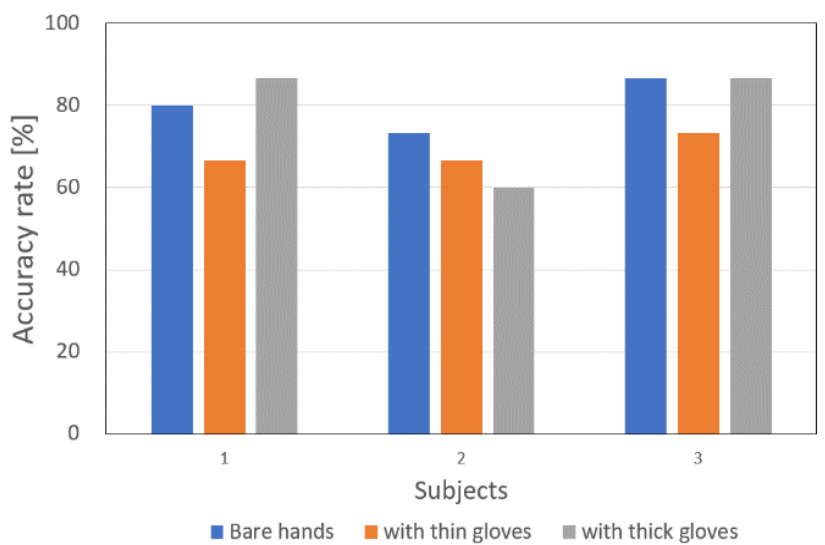

Fig.12 Experiment result

2. S Tadokoro: "Middle-Long Term Issues of Disaster Robots in Japan", Journal of the Robotics Society of Japan, Volume 32, Issue 2, Pages 154-161, 2014. (In Japanese)

3. S Tadokoro, et al.: "Overview and Outcome of WRS Disaster Robotics Category", Journal of the Robotics Society of Japan, Volume 37, Issue 3, Pages 224-234, 2019. (In Japanese)

4. N Sato, F Matsuno: "User Interface Technology for Rescue Robot Teleoperation", Journal of the Robotics Society of Japan, Volume 28, Issue 2, Pages 156-159, 2010. (In Japanese)

5. K Mima, et al.: "Tele-Manipulation with Humanoid Robot Hand/Arm via Interne", Proceedings of the Japan Joint Automatic Control Conference, Volume 54, 2K303, 2011. (In Japanese)

6. K Negishi, et al.: "i-MarioNET: Remote Operated Humanoid Robot System Controlled by Telexistence FST(Robotics for Hazardous Fields)", The Proceedings of JSME annual Conference on Robotics and Mechatronics (Robomec) 1A1-R14, 2013. (In Japanese)

7. K. Minamizawa, et al. : "A PalmWorn Haptic Display for Bimanual Operations in Virtual Environments", Proceedings of International Conference on Human Haptic Sensing and Touch Enabled Computer Applications, pp 458-463, 2008.

8. T Taniguchi, et al.: "Multi-Point Pressure Sensation Display using Pneumatic Actuators"; Proc. EuroHaptics 2019, LNCS 10894, 58-67, 2018.

9. T Okano, et al.: "Haptic Feedback for Foot Sole Using Pneumatic Pressure Device", Proc.ASIAGRAPH2016, 3 6, 2016.3..

10. Wilska, A : "On the vibrational sensitivity in different regions of the body surface", Acta Physiologica Scandinavica, vol. 31, pp. 285-289, 1954.

11. N Sato, K Ushimaru, Y Yoshifumi : " Haptic Device that Presents Sensation Corresponding to Palm on Back of Hand for Teleoperation of Robot Hand Report 1: Clarification of Required Specifications by Subject Experiment", The 38th Annual Conference of the RSJ, 2I3-03, 2020. (In Japanese) 